\title{
SPATIAL STRUCTURE OF AZIMUTHALLY SMALL-SCALE MHD WAVES IN ONE-DIMENSIONALLY INHOMOGENEOUS FINITE PRESSURE PLASMA WITH CURVED FIELD LINES
}

\author{
A.V. Petrashchuk \\ Institute of Solar-Terrestrial Physics SB RAS, \\ Irkutsk, Russia, av.petrashchuk@mail.ru
}

\author{
D.Yu. Klimushkin \\ Institute of Solar-Terrestrial Physics SB RAS, \\ Irkutsk,Russia,klimush@iszf.irk.ru
}

\begin{abstract}
We have studied propagation of hydromagnetic (MHD) waves in one-dimensionally inhomogeneous finite pressure plasma with curved field lines. Magnetic surfaces are considered to be concentric cylinders, where the cylinder's radius models the radial coordinate in Earth's magnetosphere. The waves are supposed to be azimuthally small-scale. In this approximation there are only two MHD modes - Alfvén and slow magnetosonic (SMS). We have derived an ordinary differential equation for the spatial structure of the wave field in this model. We have examined the character of the singularity on the surface of Alfvén and SMS resonances and the in-
\end{abstract}

fluence of field line curvature on them. We have determined wave transparent regions. The SMS transparent region was found to essentially broaden as compared to the straight field line case. The very existence of the Alfvén transparent region is caused by the field line curvature and finite plasma pressure; otherwise, the wave structure is represented by a localized resonance.

Keywords: MHD waves, cylinder model of the magnetosphere, MHD resonances.

\section{INTRODUCTION}

Ultra-low frequency (ULF) waves with large azimuthal wave numbers are observed in near-equatorial regions of the magnetosphere, characterized by a relatively high plasma pressure (the ratio of plasma pressure to magnetic pressure $\beta \sim 1$ ) and significant field line curvature [Agapitov, Cheremnykh, 2011; Moiseev et al., 2016; Rubtsov et al., 2018a]. These waves are generated due to injection of high-energy particles into plasma [Guglielmi, Zolotukhina, 1980; Mager, Klimushkin, 2007; Kostarev, Mager, 2017]. In recent years, azimuthally small-scale waves have been extensively studied using radars [Berngardt, 2017; Chelpanov et al., 2018] and satellites [Leonovich et al., 2015; Mager et al., 2018; Takahashi et al., 2018]. The development of the theory of such waves is a topical problem of space plasma physics because they may be responsible for the acceleration of charged particles in the magnetosphere [Ukhorskiy et al., 2009] and can serve as triggers of substorms [Rae et al., 2014].

As known, there are three MHD modes: Alfvén, fast magnetosonic (BMS), and slow magnetosonic (SMS). When accounting for plasma inhomogeneity, these modes are coupled, i.e. they cannot propagate independently of each other. The simplest model of the magnetosphere is a one-dimensionally inhomogeneous model with straight field lines, which takes into account only magnetospheric inhomogeneities across magnetic shells (box model) [Southwood, 1974; Chen, Hasegawa, 1974; Mazur, Chuiko, 2013]. This model has established the Alfvén resonance, the essence of which is as follows. Processes at the magnetospheric boundary generate a FMS wave propagating deep into the magnetosphere. On a surface located inside the magnetosphere, the FMS wave is reflected. Part of FMS energy, however, tunnels and with an exponentially decreasing amplitude propagates deeper into the magnetosphere until it reaches a magnetic surface, where its frequency coincides with the local frequency of the Alfvén wave. On a respective magnetic surface there is a sharp peak of the Alfvén wave amplitude. The azimuthal wave electric field component as well as the radial magnetic field and plasma velocity components have a logarithmic singularity; the radial electric field component and the azimuthal magnetic field and velocity components, a pole singularity.

This model in the case of finite pressure plasma has been generalized by Yumoto [1985]; the author has shown that in plasma with $\beta>0$ there should also be a resonance on a magnetic surface, where the wave frequency is equal to the local frequency of SMS waves. The singularity on this surface appeared to be the same as on the Alfvén resonant surface.

In the azimuthally small-scale limit, the FMS role can be ignored because its transparent region turns out to be localized in the immediate vicinity of the magnetopause and only an exponentially small part of FMS energy enters the magnetosphere [Guglielmi, Potapov, 1984]. Two major MHD modes in this case are Alfvén and SMS coupled due to the field line curvature [Southwood, Saunders, 1985; Walker, 1987; Cheremnykh, Parnowski, 2004]. The spatial structure of these modes in a two-dimensionally inhomogeneous magnetospheric model with irregular field line curvature has been studied in [Klimushkin, 1997; Klimushkin, 1998; Klimushkin et al., 2004]. It has been shown that in the model there are two regions of mode localization - Alfvén and SMS transparent regions. Each of them is bounded on one side by the resonant surface, where the radial wave vector component $k_{r}$ becomes infinite, and by the reflecting surface, where $k_{r}$ becomes zero. In each of the transparent regions, $k_{r}^{2}>0$. We have studied the wave field singularities on 
resonant surfaces. On the Alfvén resonant surface, the singularity was the same as in one-dimensionally inhomogeneous plasma. On the SMS resonant surface, the singularity, however, appeared to differ from that in the one-dimensionally inhomogeneous model.

It is still not quite clear to what extent we can trust the results obtained in [Klimushkin, 1997; Klimushkin, 1998; Klimushkin et al., 2004] since the mathematical methods for studying the two-dimensionally inhomogeneous magnetospheric model are not sufficiently advanced. To solve these difficulties, Cheremnykh et al. [2014, 2016] have examined the cylinder magnetospheric model, which considers magnetic surfaces to be concentric cylinders and takes into account only the inhomogeneity across the magnetic surfaces. Despite its relative simplicity, this model keeps such basic features of magnetospheric plasma as radial inhomogeneity and field line curvature. At the same time, it can avoid some mathematical difficulties specific to more realistic two-dimensionally inhomogeneous models. The cylinder model was also used to study MHD waves in the solar corona [Kaneko et al., 2015, Cheremnykh et al., 2018].

The main results of earlier studies [Klimushkin, 1997; Klimushkin, 1998, Klimushkin et al., 2004] in terms of Alfvén modes have been confirmed. Cheremnykh et al. [2014, 2016] did not, however, treat the SMS spatial structure in detail. The questions about the match between the cylinder model and the one-dimensional model of the magnetosphere studied in [Yumoto, 1985] were not addressed either. In this paper, we fill this gap.

\section{GENERAL EQUATION}

ULF waves are usually described using the system of MHD equations:

$$
\begin{aligned}
& \rho \frac{d}{d t} \vec{v}=-\nabla P+\frac{1}{c}[\vec{J} \times \vec{B}], \quad \frac{\partial \rho}{\partial t}+\nabla(\rho \vec{v})=0, \\
& J=\frac{c}{4 \pi}[\nabla \times \vec{B}], \frac{1}{c} \frac{\partial \vec{B}}{\partial t}=-[\nabla \times \vec{E}], \\
& \frac{d}{d t} \frac{P}{\rho^{\gamma}}=0, \quad \vec{E}=-\frac{1}{c}[\vec{v} \times \vec{B}],
\end{aligned}
$$

where $\vec{v}, \vec{J}, \vec{B}$ are speed, current, and magnetic field; $\rho, P$ are plasma density and pressure; $\vec{E}$ is the electric field; $\gamma$ is the heat capacity ratio, $\frac{d}{d t} \equiv \frac{\partial}{\partial t}+(\vec{v} \cdot \nabla)$. Linearizing system (1), obtain

$$
\begin{aligned}
& \rho_{0}=\frac{d \vec{v}_{1}}{\partial t}=-\nabla P_{1}+\frac{1}{c}\left(\left[\vec{J}_{1} \times \vec{B}_{0}\right]+\left[\vec{J}_{0} \times \vec{B}_{1}\right]\right), \\
& \frac{1}{c} \frac{\partial \vec{B}_{1}}{\partial t}=-\left[\nabla \times \vec{E}_{1}\right], \\
& \frac{\partial P_{1}}{\partial t}=-\left(\vec{v}_{1} \cdot \nabla\right) P_{0}-\gamma P_{0}\left(\nabla \cdot \vec{v}_{1}\right), \\
& \vec{E}_{1}=-\frac{1}{c}\left[\vec{v}_{1} \times \vec{B}_{0}\right],
\end{aligned}
$$

where quantities marked with 0 represent equilibrium values $\vec{v}, \rho, P, \vec{J}, \vec{B}, \vec{E}$; and those marked with 1 , small deviations from equilibrium.

Following [Cheremnykh et al., 2018], we consider the one-dimensionally inhomogeneous cylinder plasma model in which magnetic field lines are concentric circles and all equilibrium values depend only on the radial coordinate $r$ - circle radius (Figure 1).

The coordinate $y$, directed along the system symmetry axis plays a role of an azimuth coordinate in the magnetosphere. In this model, the magnetic field $B_{0}=\left(0, B_{0 \varphi}(r)\right.$, 0 ) and plasma pressure $P_{0}(r)$ satisfy the equilibrium condition

$$
\frac{\partial}{\partial r}\left(P_{0}+\frac{B_{0}^{2}}{8 \pi}\right)=\frac{B_{0}^{2}}{4 \pi r} .
$$

Consider a monochromatic wave with a frequency $\omega$. Due to the system symmetry in coordinates $\varphi$ and $y$, the plasma velocity can be written as

$$
\vec{v}_{1}(r, t)=\vec{v}_{1}(r) e^{i\left(-\omega t+k_{y} y+N \varphi\right)},
$$

where $k_{y}$ is the azimuthal wave vector component, $N$ is the natural number. The role of the azimuthal wave number $m$ in the cylinder model of the magnetosphere belongs to $m=k_{y} r$. The value $k_{\|}=N / r$ can be regarded as an analogue of the field-aligned wave vector component.

Then motion equation (2) can be written for the components as

$$
\begin{aligned}
& -i \rho_{0} \omega v_{1 r}=\partial_{r} \delta P-2 \frac{B_{0} B_{1 \varphi}}{4 \pi r}+i k_{\|} \frac{B_{0} B_{1 r}}{4 \pi r}, \\
& -i \rho_{0} \omega v_{1 \varphi}=-i k_{\|} \delta P+i k_{\|} \frac{B_{0} B_{1 \varphi}}{4 \pi}-\frac{B_{1 r}}{4 \pi r} \partial_{r}\left(r B_{0}\right), \\
& -i \rho_{0} \omega v_{1 y}=-i k_{y} \delta P+i \frac{k_{\|}}{r} \frac{B_{0} B_{1 y}}{4 \pi} .
\end{aligned}
$$

Here $\partial_{r}=\partial / \partial r$, and $\delta P$ is the perturbation of the total pressure (the sum of plasma and magnetic pressures):

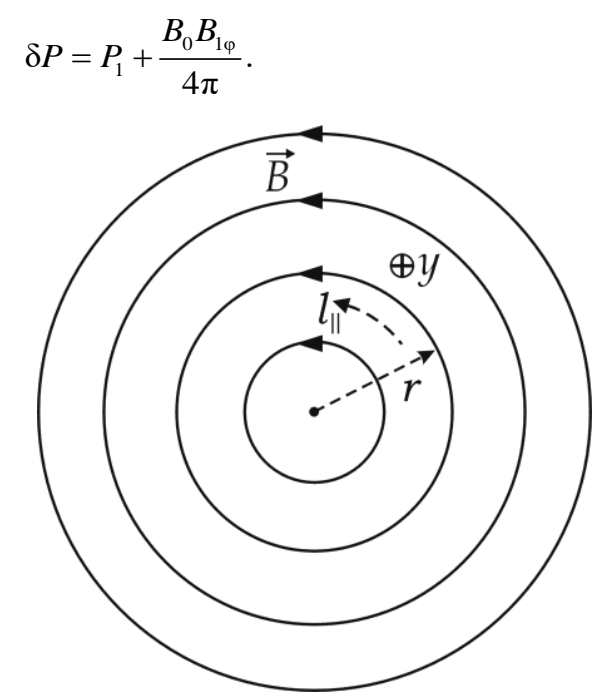

Figure 1. One-dimensionally inhomogeneous cylinder model

Equation (4) yields 


$$
\begin{aligned}
& \delta P=-i \frac{v_{1 r}}{\omega} \partial_{r} P_{0}-i \frac{\gamma P_{0}}{\omega} \times \\
& \times\left(\frac{1}{r} \partial_{r}\left(r v_{1 r}\right)+i k_{y} v_{1 y}+i k_{\|} v_{1 \varphi}\right)+\frac{B_{0} B_{1 \varphi}}{4 \pi} .
\end{aligned}
$$

After transformations in view of (7), we get:

$$
\begin{aligned}
& \delta P=\frac{\rho_{0}}{\omega}\left(v_{\mathrm{A}}^{2}+v_{\mathrm{s}}^{2}\right)\left(\frac{\omega^{2}-\omega_{\mathrm{A}}^{2}(r)}{\omega^{2}-\omega_{+}^{2}(r)}\right)\left(\frac{\omega^{2}-\omega_{\mathrm{c}}^{2}(r)}{\omega^{2}-\omega_{-}^{2}(r)}\right) \times \\
& \times\left[\frac{1}{r} \partial_{r}\left(r v_{1 r}\right)+2 \frac{v_{\mathrm{A}}^{2}}{v_{\mathrm{A}}^{2}+v_{\mathrm{s}}^{2}} \frac{v_{1 r}}{r} \frac{\omega^{2}-\omega_{\mathrm{s}}^{2}(r)}{\omega^{2}-\omega_{\mathrm{c}}^{2}(r)}\right],
\end{aligned}
$$

where $\quad v_{\mathrm{A}}=\frac{B_{0}}{\sqrt{4 \pi \rho_{0}}}, \quad v_{s}=\sqrt{\gamma \frac{P_{0}}{\rho_{0}}}, \quad v_{\mathrm{c}}=\frac{v_{\mathrm{A}} v_{\mathrm{s}}}{\sqrt{v_{\mathrm{A}}^{2}+v_{\mathrm{s}}^{2}}}$

(10) are the Alfvén, sonic, and slow magnetosonic speeds,

$$
\begin{aligned}
& \omega_{\mathrm{A}}(r)=k_{\|} v_{\mathrm{A}}, \quad \omega_{\mathrm{s}}(r)=k_{\|} v_{\mathrm{s}}, \omega_{\mathrm{c}}(r)=k_{\|} v_{\mathrm{c}}, \\
& \omega_{ \pm}^{2}(r)=\frac{\left(v_{\mathrm{A}}^{2}+v_{\mathrm{s}}^{2}\right)\left(k_{\|}^{2}+k_{y}^{2}\right)}{2} \times \\
& \times\left(1 \pm \sqrt{1-\frac{4 k_{\|}^{2} v_{\mathrm{A}}^{2} v_{\mathrm{s}}^{2}}{\left(v_{\mathrm{A}}^{2}+v_{\mathrm{s}}^{2}\right)^{2}\left(k_{\|}^{2}+k_{y}^{2}\right)}}\right) .
\end{aligned}
$$

Below we show that the frequencies $\omega_{\mathrm{A}}$ and $\omega_{\mathrm{c}}$ correspond to Alfvén and SMS resonances; frequencies $\omega_{ \pm}$, to FMS and SMS reflecting points respectively. To the azimuthally small-scale case corresponds $k_{y}>>k_{\|}$. In this case, the expressions for $\omega_{ \pm}$reduce to

$$
\begin{aligned}
& \omega_{+}^{2}=\left(v_{\mathrm{A}}^{2}+v_{\mathrm{s}}^{2}\right)\left(k_{\|}^{2}+k_{y}^{2}\right), \\
& \omega_{-}^{2}=\omega_{\mathrm{c}}^{2}\left(1+\frac{\omega_{\mathrm{c}}^{2}}{\omega_{+}^{2}}\right) .
\end{aligned}
$$

If we now substitute $\delta \mathrm{P}$ from (9) in (7), we can derive a differential equation describing the spatial structure of Alfvén, FMS, and SMS waves:

$$
\begin{aligned}
& \partial_{r}\left[\left(v_{\mathrm{A}}^{2}+v_{\mathrm{s}}^{2}\right) \frac{\left(\omega^{2}-\omega_{\mathrm{A}}^{2}\right)\left(\omega^{2}-\omega_{\mathrm{c}}^{2}\right)}{\left(\omega^{2}-\omega_{+}^{2}\right)\left(\omega^{2}-\omega_{-}^{2}\right)} \frac{\rho_{0}}{r} \partial_{r}\left(r v_{1 r}\right)\right]-2 \frac{v_{1 r}}{r} \times \\
& \times \partial_{r}\left[\rho_{0} v_{\mathrm{A}}^{2} \frac{\left(\omega^{2}-\omega_{\mathrm{A}}^{2}\right)\left(\omega^{2}-\omega_{\mathrm{s}}^{2}\right)}{\left(\omega^{2}-\omega_{+}^{2}\right)\left(\omega^{2}-\omega_{-}^{2}\right)}\right]+ \\
& +\rho_{0}\left(\omega^{2}-\omega_{\mathrm{A}}^{2}\right) v_{1 r}-2 P_{0} \chi_{\mathrm{P}} \chi_{\mathrm{c}} v_{1 r}+ \\
& +4 \frac{\rho_{0} \chi_{\mathrm{c}}^{2} \omega^{2} k_{y}^{2} v_{\mathrm{A}}^{2} v_{\mathrm{s}}^{2} v_{1 r}}{\left(\omega^{2}-\omega_{+}^{2}\right)\left(\omega^{2}-\omega_{-}^{2}\right)}=0 .
\end{aligned}
$$

Here $\chi_{\mathrm{c}}(r)=-1 / r$ is the function of field line curvature, $\chi_{\mathrm{P}}(r)=P_{0}^{-1} \partial_{r} P_{0}$ is the radial scale of plasma pressure variation. This equation coincides with that derived in [Cheremnykh et al., 2014], but is written in a more clear form.

Given the radial velocity component, from Equation (9) we can find the total pressure perturbation $\delta$ P. Next, with (7) the components $v_{1 \varphi}$ and $v_{1 y}$ are expressed through $v_{1 r}$ and $\delta P$ as follows:

$$
\begin{aligned}
& \rho_{0}\left(\omega^{2}-\omega_{\mathrm{c}}^{2}\right) v_{1 \varphi}=i k_{\|} \omega\left(\frac{v_{\mathrm{c}}^{2}}{v_{\mathrm{A}}^{2}} \delta P-2 \rho_{0} \frac{v_{\mathrm{c}}^{2}}{r} v_{1 r}\right), \\
& \rho_{0}\left(\omega^{2}-\omega_{\mathrm{c}}^{2}\right) v_{1 y}=i k_{y} \omega \delta P .
\end{aligned}
$$

Note that in the magnetosphere the component $v_{1 y} \mathrm{di}$ rected along the binormal to field lines plays a role of the azimuthal velocity component; and the component $v_{1 \varphi}$, a role of the field-aligned component. As readily seen from (15), the field-aligned velocity component in contrast to the radial and azimuthal components disappears when the plasma pressure tends to zero (i.e. when $v_{a} \rightarrow 0$ ).

\section{ONE-DIMENSIONAL CASE: NEGLIGIBLE FIELD LINE CURVATURE}

In order to compare with the results obtained by the cylinder model, we consider first the case of parallel straight field lines (box model) discussed in [Yumoto, 1985]. Given $\chi_{c}=0$, (14) yields an equation for MHD modes [Yumoto, 1985]:

$$
\begin{aligned}
& \partial_{r}\left[\left(v_{\mathrm{A}}^{2}+v_{\mathrm{s}}^{2}\right) \frac{\left(\omega^{2}-\omega_{\mathrm{A}}^{2}\right)\left(\omega^{2}-\omega_{\mathrm{c}}^{2}\right)}{\left(\omega^{2}-\omega_{+}^{2}\right)\left(\omega^{2}-\omega_{-}^{2}\right)} \rho_{0} \partial_{r} v_{1 r}\right]+ \\
& +\rho_{0}\left(\omega^{2}-\omega_{\mathrm{A}}^{2}\right) v_{1 r}=0 .
\end{aligned}
$$

In this equation there are four specific points determined by conditions

$$
\begin{array}{ll}
\omega^{2}=\omega_{\mathrm{c}}^{2}(r), & \omega^{2}=\omega_{-}^{2}(r), \\
\omega^{2}=\omega_{\mathrm{A}}^{2}(r), & \omega^{2}=\omega_{+}^{2}(r) .
\end{array}
$$

The solutions of these four equations are the points with coordinates $r_{\mathrm{c}}(\omega), r_{-}(\omega), r_{\mathrm{A}}(\omega)$, and $r_{+}(\omega)$ respectively. At small values of the parameter $\beta$ and for transversely smallscale oscillations $\left(k_{y}>>k_{\|}\right)$between respective frequencies there are relations

$$
\omega_{\mathrm{c}}<\omega_{-} \ll \omega_{\mathrm{A}} \ll \omega_{+} .
$$

In this case, throughout most of the magnetosphere the functions $\omega_{\mathrm{c}}^{2}(r), \omega_{-}^{2}(r), \omega_{\mathrm{A}}^{2}(r), \omega_{+}^{2}(r)$ are decreasing [Moore et al., 1987]. Hence, the point $r_{\mathrm{c}}$ should be the closest to Earth, followed successively by $r_{-}, r_{\mathrm{A}}$, and $r_{+}$ (Figure 2).

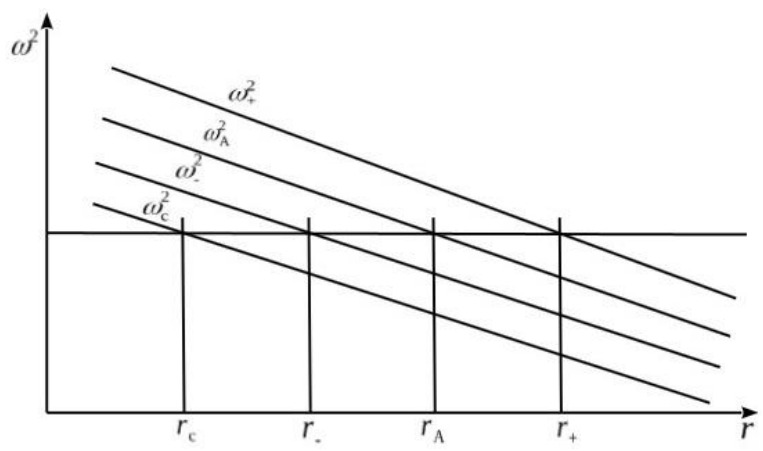

Figure 2. Graphical solution of Equations (16) and magnetic surfaces (one-dimensional model) 
First, examine the region $\left|\omega-\omega_{c}\right|, \quad\left|\omega-\omega_{-}\right| \ll \omega_{A}$, corresponding to the SMS transparent region. In this region, Equation (16) reduces to the form

$$
\partial_{r}\left[\rho_{0} \frac{\omega^{2}-\omega_{c}^{2}(r)}{\omega^{2}-\omega_{-}^{2}(r)} \partial_{r} v_{1 r}\right]-\rho_{0}\left(k_{\|}^{2}+k_{y}^{2}\right) v_{1 r}=0 .
$$

If we solve this equation in the WKB approximation, the radial wave vector component $k_{r}(r, \omega)$ will be determined from

$$
k_{r}^{2}(r, \omega)=-\left(k_{y}^{2}+k_{\| 1}^{2}\right) \frac{\omega^{2}-\omega_{-}^{2}(r)}{\omega^{2}-\omega_{\mathrm{c}}^{2}(r)} .
$$

Figure 3 plots $k_{r}^{2}(r, \omega)$ as a function of $\omega^{2}$. Formula (19) shows that at $r_{\mathrm{c}}$ and $r_{-} k_{r}$ becomes infinite and zero respectively. These points can therefore be called the SMS resonance point and the SMS reflecting point. The region between them, where $k_{r}^{2}>0$, may be referred to as the SMS transparent region.

The applicability of the WKB approximation to the SMS-wave localization region deserves a separate discussion. The WKB approximation can be used when a transparent region covers many wavelengths. A necessary (but insufficient) condition of this phenomenon is the presence of a large parameter in a wave equation. In the case of Equation (18), the large parameter is naturally considered to be $k_{y}$.

As follows from (13), the very difference $\omega_{-}^{2}-\omega_{c}^{2}$ is, however, inversely proportional to the square of this quantity:

$$
\omega_{-}^{2}-\omega_{\mathrm{c}}^{2}=\omega_{\mathrm{c}}^{2} \frac{k_{\mathrm{l}}^{2}}{k_{y}^{2}} \frac{v_{\mathrm{c}}^{2}}{v_{\mathrm{A}}^{2}+v_{\mathrm{s}}^{2}} .
$$

Thus, with increasing $k_{\mathrm{y}}$ the transparent region narrows. As readily seen, the applicability condition of the WKB approximation has the form

$$
L k_{y} \frac{k_{\|}^{2}}{k_{y}^{2}} \frac{v_{\mathrm{c}}^{2}}{v_{\mathrm{A}}^{2}+v_{\mathrm{s}}^{2}} \gg 1
$$

where $L$ is the typical scale of radially inhomogeneous plasma. Thus, the WKB approximation is applicable only to a weakly inhomogeneous plasma when $L$ is very large.

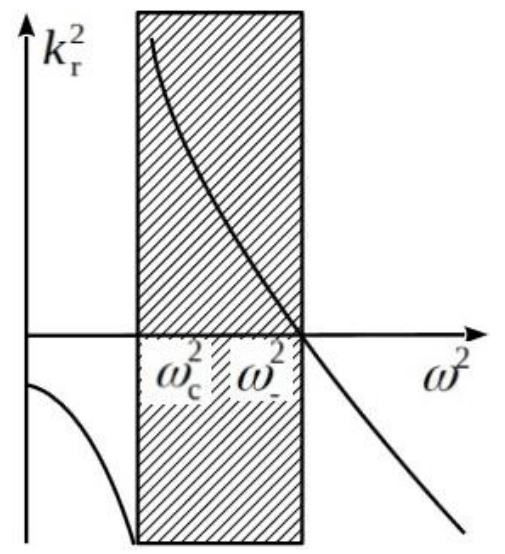

Figure 3. Squared wave vector component as a function of squared frequency in an SMS transparent region (one-dimensional model)
In the vicinity of the SMS resonance point $r \approx r_{\mathrm{c}}$, we can employ the linear expansion $\omega^{2}-\omega_{\mathrm{c}}^{2}(r)=\omega_{\mathrm{c}}^{2} \frac{r-r_{\mathrm{c}}}{l_{\mathrm{c}}}$, where $l_{\mathrm{c}}=-\frac{\omega_{\mathrm{c}}^{2}\left(r_{\mathrm{c}}\right)}{\left[\omega_{\mathrm{c}}^{2}\left(r_{\mathrm{c}}\right)\right]^{\prime}}$ is the typical scale of magnetospheric parameter variations. The $l_{\mathrm{c}}$ value is of the same order as $L$. Then, Equation (18) takes the form

$$
\partial_{r}^{2} v_{1 r}+\frac{1}{r-r_{\mathrm{c}}} \partial_{r} v_{1 r}-\frac{\lambda_{\mathrm{c}}^{-1}}{r-r_{\mathrm{c}}} v_{1 r}=0
$$

Here $\lambda_{\mathrm{c}}=\left(k_{y}^{2} l_{\mathrm{c}}\right)^{-1} \frac{\omega_{\mathrm{c}}^{2}}{\omega_{\mathrm{c}}^{2}-\omega_{-}^{2}}$ is the SMS wave length near the resonant surface (point) $r_{\mathrm{c}}$. The solution of this equation is zero-order Bessel functions

$$
v_{1 r}=v_{1} K_{0}\left(2 \sqrt{\frac{r-r_{\mathrm{c}}}{\lambda_{\mathrm{c}}}}\right)+v_{2} I_{0}\left(2 \sqrt{\frac{r-r_{\mathrm{c}}}{\lambda_{\mathrm{c}}}}\right),
$$

where $v_{1}$ and $v_{2}$ are arbitrary constants. Write the asymptotic behavior of this solution for $\left|r-r_{\mathrm{c}}\right| \ll \lambda_{\mathrm{c}}$ :

$$
v_{1 r} \sim \ln \sqrt{\frac{r-r_{\mathrm{c}}}{\lambda_{\mathrm{c}}}} .
$$

Thus, in the immediate vicinity of $r_{\mathrm{c}}$ the solution has a logarithmic singularity. This point is the SMS resonance point. When bypassing the logarithmic singularity to $r-r_{\mathrm{c}}<0$ there occurs a jump $i \pi / 2$ :

$$
v_{1 r} \sim \ln \sqrt{\frac{r-r_{\mathrm{c}}}{\lambda_{\mathrm{c}}}}-\frac{i \pi}{2} .
$$

With (15) it is easy to determine the behavior of the other two plasma velocity components too. The fieldaligned (along the external magnetic field) velocity component has a pole singularity:

$$
v_{1 \varphi}=\frac{\lambda_{\mathrm{c}}}{r-r_{\mathrm{c}}} .
$$

The same is true of the component $v_{1 y}$, directed along the binormal to magnetic shells:

$$
v_{1 y} \sim-\frac{\lambda_{\mathrm{c}}}{r-r_{\mathrm{c}}} .
$$

The electric and magnetic field components will also have the singularities:

$$
\begin{aligned}
& E_{1 r} \sim E_{0} \frac{\tilde{\lambda}_{\mathrm{c}}}{r-r_{\mathrm{c}}}, E_{1 y} \sim E_{0} \ln \sqrt{\frac{r-r_{\mathrm{c}}}{\tilde{\lambda}_{\mathrm{c}}}}, E_{1 \varphi}=0, \\
& E_{1 r}=E_{1 y}=0, E_{1 \varphi} \sim E_{0} \frac{\tilde{\lambda}_{\mathrm{c}}}{r-r_{\mathrm{c}}} .
\end{aligned}
$$

Now find the solution near the SMS reflecting point $r_{-}$when $\left|\omega-\omega_{-}\right| \ll \omega_{c}$. In this case, Equation (18) can be transformed into

$$
\partial_{r}^{2} v_{1 r}-\frac{1}{r-r_{-}} \partial_{r} v_{1 r}-\lambda_{-}^{-3}\left(r-r_{-}\right) v_{1 r}=0 .
$$

Here, it is denoted 


$$
\lambda_{-}^{-3}=\omega_{-}^{2}\left(\omega_{-}^{2}-\omega_{c}^{2}\right)^{-1} \frac{k_{y}^{2}}{l_{-}}, l_{-}=-\omega_{-}^{2} /\left[\omega_{-}^{2}\left(r_{-}\right)\right]^{\prime} . \quad \text { This }
$$

equation is for derivatives of Airy functions. Its general solution is

$$
v_{1 r}=a_{-} A i^{\prime}\left(\frac{r-r_{-}}{\lambda_{-}}\right)+b_{-} B i^{\prime}\left(\frac{r-r_{-}}{\lambda_{-}}\right) .
$$

where $a_{-}$and $b_{-}$are constants, $A i$ and $B i$ are Airy functions of the first and second kinds. Dashes denote $r$-derivatives. Thus, the presence of the singularity at $r_{-}$in wave equation (16) does not lead to a solution singularity.

In the region of Alfvén wave localization when $\omega \approx \omega_{\mathrm{A}}$ and $\omega_{\mathrm{c}} \ll \omega \ll \omega_{+}$, general equation (15) reduces to the form

$$
\begin{aligned}
& \partial_{r}\left[\omega^{2}-\omega_{\mathrm{A}}^{2}(r)\right] \partial_{r} v_{1 r}-\left(k_{y}^{2}+k_{\|}^{2}\right) \times \\
& \times\left[\omega^{2}-\omega_{\mathrm{A}}^{2}(r)\right] v_{1 r}=0 .
\end{aligned}
$$

Expanding $\omega_{\mathrm{A}}^{2}(r)$ in the neighborhood of $r \approx r_{\mathrm{A}}$ up to the first member $\omega^{2}-\omega_{\mathrm{A}}^{2}\left(r_{\mathrm{A}}\right) \approx-\left[\omega_{\mathrm{A}}^{2}\left(r_{\mathrm{A}}\right)\right]^{\prime}\left(r-r_{\mathrm{A}}\right)$, get the equation

$$
\partial_{r}^{2} v_{1 r}+\frac{1}{r-r_{\mathrm{A}}} \partial_{r} v_{1 r}-\left(k_{y}^{2}+k_{\|}^{2}\right) v_{1 r}=0
$$

representing a modified zero-order Bessel equation. Its general solution is

$$
\begin{aligned}
& v_{1 r}=v_{+} I_{0}\left(\sqrt{k_{y}^{2}+k_{\|}^{2}}\left(r-r_{\mathrm{A}}\right)\right)+ \\
& +v_{-} K_{0}\left(\sqrt{k_{y}^{2}+k_{\|}^{2}}\left(r-r_{\mathrm{A}}\right)\right),
\end{aligned}
$$

where $I_{0}(z), K_{0}(z)$ are modified Bessel functions, $v_{+}, v_{-}$ are arbitrary constants. In the vicinity of the Alfvén resonance, this solution has a logarithmic singularity

$$
v_{1 r} \sim \ln \left[\sqrt{k_{y}^{2}+k_{\|}^{2}}\left(r_{\mathrm{A}}-r\right)\right] \text {. }
$$

According to the singularity bypass rule, the analytic continuation of this solution to the region $r<r_{\mathrm{A}}$ has the form:

$$
v_{1 r} \sim \ln \left[\sqrt{k_{y}^{2}+k_{\|}^{2}}\left(r-r_{\mathrm{A}}\right)\right]-i \pi .
$$

The azimuthal velocity component $v_{1 y}$ has a pole singularity:

$$
v_{1 r} \sim \frac{1}{r-r_{\mathrm{A}}},
$$

whereas the longitudinal component $v_{1 \varphi}$ has no singularities.

Finally, at a sufficiently high frequency of $\left(\omega \gg \omega_{\mathrm{A}}\right)$ Equation (16) reduces to the form

$$
\partial_{r} \rho_{0} \frac{v_{\mathrm{A}}^{2}+v_{\mathrm{s}}^{2}}{\omega^{2}-\omega_{+}^{2}(r)} \partial_{r} v_{1 r}+\rho_{0} v_{1 r}=0 \text {. }
$$

When this equation is solved in the WKB approximation, the squared radial wave vector component is defined by the expression

$$
k_{r}^{2}=\frac{\omega^{2}}{v_{\mathrm{A}}^{2}+v_{\mathrm{s}}^{2}}-\left(k_{y}^{2}+k_{\|}^{2}\right) .
$$

This expression is derived directly from the dispersion equation for FMS [Southwood, 1974]. From (32) we can see that the point $r_{+}$, where the equality $\omega^{2}-\omega_{+}^{2}(r)=0$ holds, is the FMS reflecting point because at this point the radial wave vector component becomes zero.

Examine the solution near this point in more detail. Expanding $\omega_{+}^{2}(r)$ in series in $r \approx r_{+}$,

$$
\omega^{2}-\omega_{+}^{2}(r) \approx-\left[\omega_{+}^{2}\left(r_{+}\right)\right]^{\prime}\left(r-r_{+}\right)=\omega_{+}^{2} \frac{r-r_{+}}{l_{+}},
$$

get

$$
\partial_{r}^{2} v_{1 r}-\frac{1}{r-r_{+}} \partial_{r} v_{1 r}+\lambda_{+}^{-3}\left(r-r_{+}\right) v_{1 r}=0,
$$

where $\lambda_{+}^{-3}=l_{+}^{-1} \frac{\omega_{+}^{2}}{v_{\mathrm{A}}^{2}+v_{\mathrm{s}}^{2}}$. A solution of (33) will be

$$
v_{1 r}=a_{+} A i^{\prime}\left(-\frac{r-r_{+}}{\lambda_{+}}\right)+b_{+} B i^{\prime}\left(-\frac{r-r_{+}}{\lambda_{+}}\right) \text {. }
$$

This solution is regular. As in the case of the SMS reflecting point, the presence of the singularity in the wave equation does not lead to a solution singularity.

\section{AZIMUTHALLY}

\section{SMALL-SCALE WAVES IN THE CYLINDER MODEL}

Cheremnykh et al. [2014] have derived a differential equation used to find the spatial structure of SMS and Alfvén modes. The question about the SMS spatial structure was not, however, completely answered. Address this question in more detail.

In the azimuthally small-scale case (when $k_{y} \gg k_{\|}$), the wave frequency is much smaller than the FMS reflecting one: $\omega \ll \omega_{+}$. Then, differential equation (14) takes the form

$$
\begin{aligned}
& -\partial_{r}\left[\frac{\left(\omega^{2}-\omega_{\mathrm{A}}^{2}\right)\left(\omega^{2}-\omega_{\mathrm{c}}^{2}\right)}{\omega^{2}-\omega_{-}^{2}}\right] \frac{\rho_{0}}{r} \partial_{r}\left(r v_{1 r}\right)+ \\
& +\frac{2}{r} \frac{v_{\mathrm{A}}^{2}}{v_{\mathrm{A}}^{2}+v_{\mathrm{s}}^{2}} v_{1 r} \partial_{r}\left[\rho_{0} \frac{\left(\omega^{2}-\omega_{\mathrm{A}}^{2}\right)\left(\omega^{2}-\omega_{\mathrm{s}}^{2}\right)}{\omega^{2}-\omega_{-}^{2}}\right]+ \\
& +k_{y}^{2} \frac{\left(\omega^{2}-\omega_{1}^{2}\right)\left(\omega^{2}-\omega_{2}^{2}\right)}{\omega^{2}-\omega_{-}^{2}} v_{1 r}=0 .
\end{aligned}
$$

Here $\omega_{1,2}$ is the solution of the biquadratic equation

$$
\left(\omega^{2}-\omega_{\mathrm{A}}^{2}-2 P_{0} \frac{\chi_{\mathrm{p}} \chi_{\mathrm{c}}}{\rho_{0}}\right)\left(\omega^{2}-\omega_{-}^{2}\right)-4 \chi_{\mathrm{c}}^{2} \omega^{2} v_{\mathrm{c}}^{2}=0 .
$$

Write an expression for $\omega_{1.2}$ :

$$
\begin{aligned}
& \omega_{1,2}^{2}=\frac{1}{2}\left[\left(\omega_{\mathrm{p}}^{2}+\omega_{-}^{2} 4 v_{\mathrm{c}}^{2} \chi_{\mathrm{c}}^{2}\right) \pm\right. \\
& \left. \pm \sqrt{\left(\omega_{\mathrm{p}}^{2}-\omega_{-}^{2}\right)^{2}+8\left(\omega_{\mathrm{p}}^{2}+\omega_{-}^{2}\right) v_{\mathrm{c}}^{2} \chi_{\mathrm{c}}^{2}+16 v_{\mathrm{c}}^{4} \chi_{\mathrm{c}}^{4}}\right]
\end{aligned}
$$


where it is denoted

$$
\omega_{\mathrm{p}}^{2}=\omega_{\mathrm{A}}^{2}+\left(2 P_{0} / \rho_{0}\right) \chi_{\mathrm{p}} \chi_{\mathrm{c}} .
$$

Expression (35) is in complete agreement with the equation obtained in [Cheremnykh et al., 2014] for coupled Alfvén and SMS modes.

If the plasma pressure is not very high and the condition $\beta\left(\chi_{\mathrm{c}} / k_{\mathrm{t}}\right)^{2} \ll 1$, holds, the frequencies $\omega_{1,2}$ can be written in the approximate form:

$$
\begin{aligned}
& \omega_{1}^{2}=\omega_{\mathrm{c}}^{2}\left(1-4 \frac{\chi_{\mathrm{c}}^{2} v_{\mathrm{c}}^{2}}{\omega_{\mathrm{A}}^{2}} \chi_{\mathrm{p}}\right), \\
& \omega_{2}^{2}=\omega_{\mathrm{A}}^{2}+4 \chi_{\mathrm{c}}^{2} v_{\mathrm{c}}^{2}-\beta v_{\mathrm{A}}^{2} \chi_{\mathrm{c}} \chi_{\mathrm{p}}=\omega_{\mathrm{p}}^{2}-4 \chi_{\mathrm{c}}^{2} v_{\mathrm{c}}^{2} .
\end{aligned}
$$

Hence, the frequency $\omega_{1}$ is closer to the SMS resonant frequency $\omega_{c}$; and $\omega_{2}$, to the Alfvén resonant frequency $\omega_{\mathrm{A}}$. From these formulas we can also see that the frequency $\omega_{1}$ has always been lower than the SMS resonant one, $\omega_{1}<\omega_{\mathrm{c}}$. However, the frequency $\omega_{2}$ may be both higher and lower than the Alfvén resonant frequency.

First, we consider the solution of this equation in the WKB approximation. The squared radial wave vector component is determined from the relation

$$
k_{r}^{2}(r, \omega)=-k_{y}^{2} \frac{\left[\omega^{2}-\omega_{1}^{2}(r)\right]}{\left[\omega^{2}-\omega_{\mathrm{c}}^{2}(r)\right]} \frac{\left[\omega^{2}-\omega_{2}^{2}(r)\right]}{\left[\omega^{2}-\omega_{\mathrm{A}}^{2}(r)\right]} .
$$

As we can see, when equalities

$$
\omega=\omega_{1}(r), \omega=\omega_{2}(r)
$$

hold, the radial wave vector component becomes zero. Hence, the frequencies $\omega_{1}$ and $\omega_{2}$ represent reflecting frequencies of SMS and Alfvén modes respectively. The points $r_{1}$ and $r_{2}$ as solutions of (39) will be called reflecting points of SMS and Alfvén modes respectively. Since throughout most of the magnetosphere the functions $\omega_{\mathrm{c}}(r), \omega_{\mathrm{A}}(r)$, and $\omega_{1,2}(r)$ are decreasing, there is always the inequality $r_{1}<r_{\mathrm{c}}$. In the case of $\omega_{\mathrm{A}}<2, r_{\mathrm{A}}<r_{2}$; in the opposite case of $\omega_{\mathrm{A}}>\omega_{2}, r_{\mathrm{A}}>r_{2}$ (Figure 4).

Figure 5 plots the squared radial wave vector component $k_{r}^{2}(r)$ as a function of the squared frequency $\omega^{2}$. Panel $a$ corresponds to $\omega_{\mathrm{A}}<\omega_{2}$; panel $b$, to $\omega_{\mathrm{A}}>\omega_{2}$.

Numerals I and II denote areas of propagation of SMS and Alfvén waves respectively. Numeral III refers to frequencies at which wave propagation is impossible. Accordingly, the SMS transparent region is in the range $r_{1}<r<r_{\mathrm{c}}$; the Alfvén mode transparent region, in the range $r_{\mathrm{A}}<r<r_{2}(a)$ and $r_{2}<r<r_{\mathrm{A}}(b)$.

For the Alfvén transparent region the resonant surface $r_{\mathrm{A}}$ is also referred to as the toroidal surface; the reflecting surface $r_{2}$, as the poloidal surface [Leonovich, Mazur, 1993]. When $k_{r} \rightarrow \infty$, field lines seem to slide over unperturbed magnetic surfaces (cylinders in our case); and when $k_{r}=0$ they oscillate along the normal to magnetic shells.

If an opacity region is much wider than the transparent regions of each of the modes, Equation (35) allows for a further simplification in the Alfvén and SMS transparent regions. Consider the Alfvén transparent region in the case of $\left|\omega_{\mathrm{A}}-\omega_{2}\right| \ll \omega$ and $\omega_{\mathrm{A}}, \omega_{2} \gg \omega_{\mathrm{c}}, \omega_{1}$ for large values of $k_{y}$. Then Equation (35) reduces to the

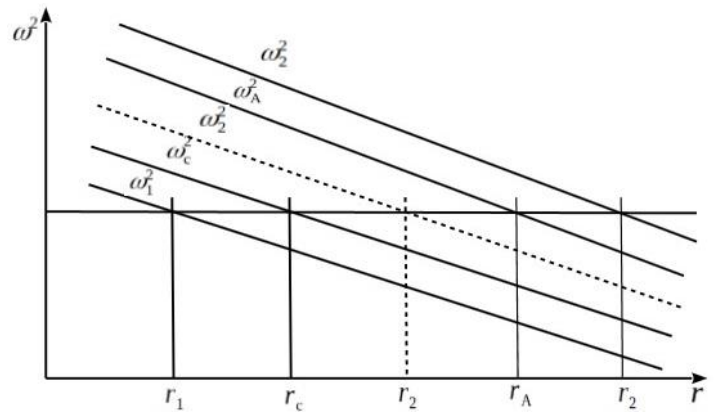

Figure 4. Graphical solution of Equations (40) and magnetic surfaces $r_{\mathrm{c}}(\omega), r_{1}(\omega), r_{\mathrm{A}}(\omega), r_{2}(\omega)$ (the last one is shown in two cases: $\omega_{\mathrm{A}}<\omega_{2}$ and $\left.\omega_{\mathrm{A}}>\omega_{2}\right)$

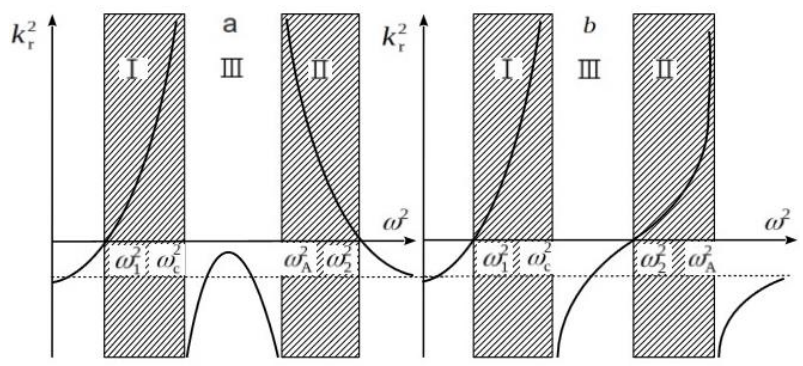

Figure 5. Squared radial wave vector component as a function of squared frequency $\omega^{2}$. When $\omega \approx \omega,{ }_{A}$ and $\omega \approx \omega_{\mathrm{c}} k_{r}^{2}(r)$ have a discontinuity from $-\infty$ to $\infty$, and when passing through points $\omega_{1}^{2}$ and $\omega_{2}^{2}$ the function $k_{r}^{2}(r)$ changes sign $k_{r}^{2}(r)<0$

form

$$
-\partial_{r}\left[\omega^{2}-\omega_{\mathrm{A}}^{2}(r)\right] \partial_{r} v_{1 r}+k_{y}^{2}\left[\omega^{2}-\omega_{2}^{2}(r)\right] v_{1 r}=0,
$$

which coincides with the equation for the Alfvén mode derived in [Cheremnykh et al., 2014]. Since the authors have carried out a sufficiently detailed analysis of the solution of this equation, we do not dwell on it. We note only that near the resonant surface there is still the logarithmic singularity of the wave field as in the one-dimensional case:

$$
v_{1 r} \sim \ln \sqrt{\frac{r-r_{\mathrm{A}}}{\lambda_{\mathrm{A}}}}, \quad v_{1 \varphi} \sim \ln \sqrt{\frac{r-r_{\mathrm{A}}}{\lambda_{\mathrm{A}}}},
$$

where

$\lambda_{\mathrm{A}}=k_{y}^{2} l_{\mathrm{A}}\left(\omega_{\mathrm{A}}^{2}-\omega_{2}^{2}\right) / \omega_{\mathrm{A}}^{2}, \quad l_{\mathrm{A}}=-\omega_{\mathrm{A}}^{2} /\left[\omega_{\mathrm{A}}^{2}\left(r_{\mathrm{A}}\right)\right]^{\prime}$. The azimuthal velocity component $v_{1 y}$ takes the form

$$
v_{1 y} \sim \frac{1}{r-r_{\mathrm{A}}} \text {. }
$$
form

The electric and magnetic field components have the

$$
\begin{aligned}
& E_{1 r} \sim E_{0} \frac{\lambda_{A}}{r-r_{\mathrm{A}}}, E_{1 y} \sim E_{0} \ln \sqrt{\frac{r-r_{\mathrm{A}}}{\lambda_{\mathrm{A}}}}, E_{1 \varphi}=0, \\
& E_{1 r}=E_{1 y}=0, E_{1 \varphi} \sim E_{0} \frac{\lambda_{\mathrm{A}}}{r-r_{\mathrm{A}}} .
\end{aligned}
$$

Away from the resonant surface, the behavior of the 
wave field appears, however, to be different than in the one-dimensional case. If in the one-dimensional case the Alfvén wave is an isolated resonant peak, in the curved geometry the solution near the resonance point is oscillatory, with the wavelength decreasing as it approaches the singularity. This is also consistent with the results obtained in [Klimushkin, 1997; Klimushkin, 1998; Klimushkin et al., 2004] for the two-dimensionally inhomogeneous magnetospheric model with variable field line curvature and longitudinal inhomogeneous plasma.

In this paper, we focus mainly on the SMS transparent region. Consider the limiting case $\left|\omega_{\mathrm{c}}-\omega_{1}\right| \ll \omega$ and $\omega_{\mathrm{c}}, \omega_{1} \ll \omega_{\mathrm{A}}, \omega_{2}$. In this case, Equation (35) reduces to the form

$$
\begin{aligned}
& \partial_{r}\left[\frac{\omega^{2}-\omega_{\mathrm{c}}^{2}(r)}{\omega^{2}-\omega_{-}^{2}(r)}\right] \partial_{r} v_{1 r}-\frac{2}{r} \frac{v_{\mathrm{A}}^{2}}{v_{\mathrm{A}}^{2}+v_{\mathrm{s}}^{2}} \times \\
& \times v_{1 r} \partial_{r}\left[\frac{\omega^{2}-\omega_{\mathrm{s}}^{2}(r)}{\omega^{2}-\omega_{-}^{2}(r)}\right]-k_{y}^{2} \frac{\omega^{2}-\omega_{1}^{2}(r)}{\omega^{2}-\omega_{-}^{2}(r)} v_{1 r}=0 .
\end{aligned}
$$

Examine the vicinity of $r_{\mathrm{c}}$ : $\left|\omega-\omega_{c}\right| \ll\left|\omega-\omega_{-}\right|,\left|\omega-\omega_{1}\right|$. Then Equation (42) allows for a further simplification:

$$
\partial_{r}\left[\omega^{2}-\omega_{\mathrm{c}}^{2}(r)\right] \partial_{r} v_{1 r}-k_{y}^{2}\left[\omega_{\mathrm{c}}^{2}-\omega_{1}^{2}(r)\right] v_{1 r}=0 .
$$

Near the resonance point $r \approx r_{\mathrm{c}}$ we can expand $\omega_{\mathrm{c}}(r)$ in a power series

$$
\omega^{2}-\omega_{\mathrm{c}}^{2}(r) \approx-\left(\omega_{\mathrm{c}}^{2}\right)^{\prime}\left(r-r_{\mathrm{c}}\right)=\omega_{\mathrm{c}}^{2} \frac{r-r_{\mathrm{c}}}{l_{\mathrm{c}}} \text {. Then dif- }
$$

ferential equation (43) becomes

$$
\partial_{r} \omega_{\mathrm{c}}^{2} \frac{r-r_{\mathrm{c}}}{l_{\mathrm{c}}} \partial_{r} v_{1 r}-k_{y}^{2}\left(\omega_{\mathrm{c}}^{2}-\omega_{1}^{2}\right) v_{1 r}=0 .
$$

The solution of this equation has the form

$$
v_{1 r}=\tilde{v}_{1} K_{0}\left(\sqrt{\frac{r-r_{\mathrm{c}}}{\lambda_{\mathrm{c}}}}\right)+\tilde{v}_{2} I_{0}\left(\sqrt{\frac{r-r_{\mathrm{c}}}{\lambda_{\mathrm{c}}}}\right) \text {, }
$$

where it is denoted $\lambda_{\mathrm{c}}^{-1}=k_{y}^{2} l_{\mathrm{c}} \frac{\omega_{\mathrm{c}}^{2}-\omega_{1}^{2}}{\omega_{\mathrm{c}}^{2}}$.

Given $\left|r-r_{\mathrm{c}}\right| \ll \lambda_{\mathrm{c}}$ the solution of (45) has the same logarithmic singularity as in (23):

$$
v_{1 r} \sim \ln \sqrt{\frac{r-r_{\mathrm{c}}}{\lambda_{\mathrm{c}}}} .
$$

The field-aligned and azimuthal components of the displacement have a pole singularity:

$$
v_{1 \varphi} \sim \frac{\lambda_{\mathrm{c}}}{r-r_{\mathrm{c}}}, \quad v_{1 y} \sim \frac{\lambda_{\mathrm{c}}}{r-r_{\mathrm{c}}} .
$$

Sets of electric and magnetic fields have the form

$$
\begin{aligned}
& E_{1 r} \sim B_{0} \frac{\tilde{\lambda}_{c}}{r-r_{c}}, E_{1 y} \sim B_{0} \ln \sqrt{\frac{r-r_{c}}{\tilde{\lambda}_{c}}}, E_{1 \varphi}=0, \\
& B_{1 r}=B_{1 y}=0, B_{1 \varphi} \sim B_{0} \frac{\tilde{\lambda}_{A}}{r-r_{c}} .
\end{aligned}
$$

The analytic continuation of this solution is:

$v_{1 r} \sim \ln \sqrt{\frac{r_{\mathrm{c}}-r}{\lambda_{\mathrm{c}}}}-i \frac{\pi}{2}$.

Consider now the wave field structure near the other boundary of the SMS transparent region - the turning point $r_{1}$. To do this, put $\left|\omega-\omega_{1}\right| \ll\left|\omega-\omega_{-}\right|,\left|\omega-\omega_{c}\right|$ in Equation (42). We obtain an equation in the azimuthally small-scale approximation

$$
\partial_{r}^{2} v_{1 r}+k_{y}^{2} \frac{\omega^{2}-\omega_{1}^{2}(r)}{\omega_{c}^{2}-\omega_{-}^{2}(r)} v_{1 r}=0 .
$$

Using the linear expansion of the function $\omega_{1}^{2}(r)$ near the point

$$
r \approx r_{1}, \quad \omega^{2}-\omega_{1}^{2}(r) \approx-\left[\omega_{1}^{2}\left(r_{1}\right)\right]^{\prime}\left(r-r_{1}\right)=\omega_{1}^{2} \frac{r-r_{1}}{l_{1}},
$$

we obtain an Airy equation

$$
\partial_{r}^{2} v_{1 r}+\lambda_{1}^{-3}\left(r-r_{1}\right) v_{1 r}=0
$$

Where $\lambda_{1}^{-3}=k_{y}^{2} \omega_{1}^{2} / l_{1}\left(\omega_{\mathrm{c}}^{2}-\omega_{-}^{2}\right)$. Find solutions of (49):

$$
v_{1 r}=a_{1} A i\left(-\lambda_{1}^{-1}\left(r-r_{1}\right)\right)+b_{1} B i\left(-\lambda_{1}^{-1}\left(r-r_{1}\right)\right) \text {. }
$$

As for the point $r_{-}$(the turning point in the one-dimensional case), it is still a singularity of the equation for SMS and in the curved case, but its meaning changes. In the vicinity of this point, where $\left|\omega-\omega_{-}\right| \gg\left|\omega-\omega_{c}\right|,\left|\omega-\omega_{1}\right|$, Equation (35) allows for a further simplification:

$$
\begin{aligned}
& -\partial_{r} \frac{\omega_{-}^{2}-\omega_{\mathrm{c}}^{2}(r)}{\omega^{2}-\omega_{-}^{2}(r)} \partial_{r} v_{1 r}+\frac{2}{r} \frac{v_{\mathrm{A}}^{2}}{v_{\mathrm{A}}^{2}+v_{\mathrm{s}}^{2}} v_{1 r} \partial_{r}\left[\frac{\omega_{-}^{2}-\omega_{\mathrm{s}}^{2}}{\omega^{2}-\omega_{-}^{2}}\right]+ \\
& +k_{y}^{2} \frac{\omega^{2}-\omega_{1}^{2}(r)}{\omega^{2}-\omega_{-}^{2}(r)} v_{1 r}=0 .
\end{aligned}
$$

If we linearly expand $\omega_{-}(r)$ near the point $r \approx r_{-}$, Equation (51) can be transformed into

$$
\frac{1}{r-r_{-}} \partial_{r}^{2} v_{1 r}-\frac{1}{\left(r-r_{-}\right)^{2}} \partial_{r} v_{1 r}-\frac{\lambda_{-}^{-1}}{\left(r-r_{-}\right)^{2}} v_{1 r}=0 .
$$

Here it is denoted

$$
\lambda_{-}^{-1}=\frac{2}{r} \frac{v_{\mathrm{A}}^{2}}{v_{\mathrm{A}}^{2}+v_{\mathrm{s}}^{2}}\left[\frac{\omega_{-}^{2}-\omega_{\mathrm{s}}^{2}}{\omega_{-}^{2}-\omega_{\mathrm{c}}^{2}}\right] .
$$

The general solution of (52) has the form

$$
\begin{aligned}
& v_{1 r}=\left(r-r_{-}\right) \times \\
& \times\left[a J_{2}\left(2 \sqrt{-\frac{r-r_{-}}{\lambda_{-}}}\right)+b Y_{2}\left(2 \sqrt{-\frac{r-r_{-}}{\lambda_{-}}}\right)\right],
\end{aligned}
$$

where $J_{2}(z), Y_{2}(z)$ are second-order Bessel functions; $a, b$ are arbitrary constants.

$$
\text { If } r \rightarrow r \text {, }
$$

$v_{1 r} \sim a\left(r-r_{-}\right)+b\left[-\frac{1}{\pi}+\frac{r-r_{-}}{2 \pi \lambda_{-}}\left(\ln \sqrt{\frac{r-r_{-}}{2 \lambda_{-}}}-i \frac{\pi}{2}\right)\right]$. 
Thus, as the point $r_{-}$is approached the wave amplitude in the radial displacement component $v_{1 r}$ tends to a finite value. In the azimuthal component, the wave has, however, a logarithmic singularity

$$
v_{1 y} \sim \frac{b}{2 \pi \lambda_{-}}\left(\ln \sqrt{\frac{r-r_{-}}{2 \lambda_{-}}}-i \frac{\pi}{2}\right) .
$$

The azimuthal and field-aligned components of electric and magnetic fields take the form

$$
\begin{aligned}
& E_{1 r} \sim B_{0} \frac{b}{2 \pi \tilde{\lambda}_{-}}\left(\ln \sqrt{\frac{r-r_{-}}{2 \tilde{\lambda}_{-}}}-i \frac{\pi}{2}\right), \\
& E_{1 y} \sim B_{0} \frac{r-r_{-}}{2 \pi \tilde{\lambda}_{-}}\left(\ln \sqrt{\frac{r-r_{-}}{2 \tilde{\lambda}_{-}}}-i \frac{\pi}{2}\right), \\
& E_{1 \varphi}=0, B_{1 r}=B_{1 y}=0, \\
& B_{1 \varphi} \sim B_{0} \frac{b}{2 \pi \tilde{\lambda}_{-}}\left(\ln \sqrt{\frac{r-r_{-}}{2 \tilde{\lambda}_{-}}}-i \frac{\pi}{2}\right) .
\end{aligned}
$$

Thus, if in the one-dimensional model the point $r_{-}$represents a reflecting point, in the curved model it is a point of the secondary (logarithmic) resonance. There is no analogue of this point in the one-dimensional model.

\section{DISCUSSION}

Compare results of the analysis of the cylinder model with those of the one-dimensional model discussed in Section 2 and with those of the dipole model studied in [Klimushkin, 1997; Klimushkin, 1998; Klimushkin et al., 2004].

First, examine the Alfvén mode. As we saw in Section 2 , in the one-dimensional case the Alfvén mode unlike SMS has no reflecting surface. Since the difference $\omega_{\mathrm{A}}^{2}-\omega_{2}^{2}$ is proportional to the plasma pressure, in the cylinder model, but with a cold plasma, the Alfvén mode has no reflecting surface either. Thus, for the Alfvén mode the reflecting surface exists only in the finite pressure plasma with curved field lines. The resonant singularity is logarithmic as in the one-dimensional model, but the phase jumps by $\pi / 2$, not by $\pi$ as in the one-dimensional model. All these results check well with the results obtained in the dipole model of the magnetosphere, which takes into account inhomogeneous plasma and magnetic field along field lines [Klimushkin, 1997; Klimushkin, 1998; Klimushkin et al., 2004].

The situation with SMS is somewhat different. In the one-dimensional case, the SMS reflecting surface was determined by $\omega=\omega_{-}(r)$ and was located more to the right of the SMS resonant surface. In this case, as the azimuthal wave vector component $k_{y}$ increased, the SMS transparent region narrowed. In the cylinder case, the SMS reflecting surface was defined by $\omega=\omega_{1}(r)$. In this case, it is located more to the left of the SMS resonant surface. The difference between the SMS reflecting frequency and the respective resonant frequency is determined only by plasma and magnetic field parameters and does not depend on $k_{\mathrm{y}}$. Thus, the SMS transparent region in the cylinder model is much wider than in the one-dimensional model. This result agrees with the results of the dipole model [Klimushkin, 1997; Klimushkin, 1998].

The behavior of the resonant singularity in SMS in the one-dimensional and cylinder models is the same. In this case, the dipole model gives different results [Klimushkin, 1997; Klimushkin, 1998], which is most likely to be its artifact. Indeed, let us turn again to Equation (42) for SMS. As already noted, the difference between $\omega_{c}$ and $\omega_{-}$in the azimuthally small-scale limit tends to zero. If we also neglect the differences between $\omega_{\mathrm{c}}$ and $\omega_{\mathrm{s}}$, in the azimuthally small-scale limit the second term of this equation seems to be ignored as well. Then it takes the form

$$
\partial_{r} \partial_{r} v_{1 r}-k_{y}^{2} \frac{\omega^{2}-\omega_{1}^{2}(r)}{\omega^{2}-\omega_{\mathrm{c}}^{2}(r)} v_{1 r}=0,
$$

which coincides with the equation for SMS obtained by the dipole model in [Klimushkin, 1997; Klimushkin, 1998]. Obviously, the resonant singularity in Equation (55) has the form $v_{1 r} \sim\left(r-r_{c}\right) \ln \left(r-r_{c}\right)$ which differs from the correct result of (46) $v_{1 r} \sim \ln \left(r-r_{c}\right)$. In addition, Equation (56) does not contain the secondary (logarithmic) resonance. Thus, we can conclude that the methods of studying azimuthally small-scale waves in the dipole model used in [Klimushkin, 1997; Klimushkin, 1998] are too crude to examine the behavior of SMS near the resonant surface, although they are quite suitable for studying the mode near the reflecting surface.

It should be noted that there are two questions concerning the subject matter of this work, which we did not address in this paper. The first of them is the ballooning instability whose development requires curved field lines and finite pressure plasma [Burdo et al., 2000; Agapitov et al., 2006; Liu, 1997; Bhattacharjee et al., 1998; Golovchanskaya et al., 2006]. This instability occurs in the SMS branch of oscillations during a sharp decrease in pressure with distance from Earth [Cheremnykh, Parnowski, 2004; Mazur et al., 2012; Rubtsov et al., 2018b]. In this paper, we treat modes resistant to ballooning perturbations. In addition, in a collisionless plasma (plasma of Earth's magnetosphere) the correct consideration of the finite pressure is possible only within the framework of kinetics when in the plasma there may exist ULF modes that do not occur in MHD, such as drift-compressional and mirror modes [Mikhailovskii, Fridman, 1966; Hasegawa, 1969; Rosenbluth, 1981]. In an inhomogeneous plasma, these modes are coupled with the Alfvén ones [Chen, Hasegawa, 1991]. This coupling has been studied using the cylinder model in [Pokhotelov et al., 1985; Woch et al., 1988; Klimushkin et al., 2012]; using a more realistic dipole model, in [Mager, Klimushkin, 2017].

\section{CONCLUSION}

The analysis of the cylinder model for one-dimensionally inhomogeneous finite pressure plasma with curved field lines enables us to do the following.

1. To derive an ordinary differential equation describing the transverse structure of Alfvén, FMS, and SMS modes. 
Using the WKB approximation, we have determined transparent regions of these modes.

2. To examine singularities on magnetic shells of Alfvén and SMS resonances in terms of field line curvature and finite pressure. In the one-dimensional case, the Alfvén wave is an isolated resonant peak, and in the curved geometry the solution near the resonance point is oscillatory, whereas the behavior of the resonant singularity of SMS in the one-dimensional and cylinder model is the same. The behavior of the SMS resonant singularity in the cylinder model differs from that in the dipole model in [Klimushkin, 1997; Klimushkin, 1998].

3. To show that the SMS behavior near the reflecting surface $\omega=\omega_{1}(r)$ in the cylinder model coincides with the behavior in the dipole model. As for the turning point in the one-dimensional case $\omega=\omega(r)$, in terms of field line curvature it becomes a point of the secondary (logarithmic) resonance for SMS.

The work was performed with budgetary funding of Basic Research program II.12. We are grateful to P.N. Mager for valuable comments and suggestions.

\section{REFERENCES}

Agapitov A.V., Cheremnykh O.K. Polarization of ULF waves in the Earth's magnetosphere. Kinematics and Physics of Celestial Bodies. 2011, vol. 27, no. 3, pp. 117-123. DOI: 10.3103/S0884591311030020.

Agapitov A.V., Parnowski A.S., Cheremnykh O.K. Spectrum of transversally small-scale perturbations in the inner Earth's magnetosphere. Kinematika i Fizika Nebesnykh Tel. [Kinematics and Physics of Celestial Bodies]. 2006. vol. 22, no. 6, pp. 387-401. (In Russian).

Berngardt O., Space weather impact on radio device operation. Solar-Terr. Phys. 2017, vol. 3, no. 3, pp. 37-53. DOI 10.12737/stp-33201705.

Bhattacharjee A., Ma Z., Wang X. Dynamics of thin current sheets and their disruption by ballooning instabilities: A mechanism for magnetospheric substorms. Physics of Plasmas. 1998, vol. 5, pp. 2001-2009. DOI: 10.1063/1.872871.

Burdo O.S., Cheremnykh O.K., Verkhoglyadova O.P. Study of ballooning modes in the inner magnetosphere of the Earth. Izvestiya Akademii nauk. Fizika [Bulletin of the Russian Academy of Sciences: Physics]. 2000, vol. 64, pp. 1896-1900. (In Russian).

Chen L., Hasegawa A. A theory of long period magnetic pulsations. 1. Steady state excitation of field line resonance. $J$. Geophys. Res. 1974, vol. 79, iss. 7, pp. 1024-1032. DOI: 10.1029/ JA079i007p01024.

Chen L., Hasegawa A. Kinetic theory of geomagnetic pulsations: 1. Internal excitations by energetic particles. $J$. Geophys. Res. 1991, vol. 96, pp. 1503-1512. DOI: 10.1029/ 90JA02346.

Chelpanov M.A., Mager O.V., Klimushkin D.Yu., Berngardt O.I., Mager O.V. Experimental evidence of drift compressional waves in the magnetosphere: An Ekaterinburg coherent decameter radar case study. J. Geophys. Res.: Space Phys. 2016. V. 121. P. 1315-1326. DOI: 10.1002/2015JA022155.

Chelpanov M.A., Mager O.V., Mager P.N., Klimushkin D.Yu., Berngardt O.I. Properties of frequency distribution of Pc5-range pulsations observed with the Ekaterinburg decameter radar in the nightside ionosphere. J. Atmos. Sol.-Terr. Phys. 2018, vol. 167, pp. 177-183. DOI: 10.1016/j.jastp.2017.12.002.

Cheremnykh O.K., Parnowski A.S. The theory of ballooning perturbations in the inner magnetosphere of the Earth. $A d v$.
Space Res. 2004, vol. 33, pp. 769-773.

Cheremnykh O.K., Klimushkin D., Kostarev D.V. On the structure of azimuthally small-scale ULF oscillations of hot space plasma in a curved magnetic field. Modes with continuous spectrum. Kinematics and Physics of Celestrial Bodies. 2014, vol. 30, no. 5, pp. 209-222.

Cheremnykh O.K., Klimushkin D.Yu., Mager P.N. On the Structure of Azimuthally Small-Scale ULF Oscillations of a Hot Space Plasma in a Curved Magnetic Field: Modes with Discrete Spectra. Kinematics and Physics of Celestial Bodies. 2016, vol. 32, no. 3, pp. 120-128.

Cheremnykh O.K., Kryshtal A.N., Tkachenko A.A. Kink mode $\mathrm{m}=1$ in magnetic tube with discontinuous magnetic field. $A d v$. Space Res. 2018, vol. 61, pp. 603-610. DOI: 10.1016/j.asr.2017.05.026.

Guglielmi A.V., Zolotukhina N.A. Excitation of Alfvén oscillations of the magnetosphere by the asymmetric ring current. Issledobaniya po geomagnetizmu, aeronomii i fizike Solntsa [Res. on Geomagnetism, Aeronomy and Solar Physics]. 1980, vol. 50, pp. 129-137. (In Russian).

Guglielmi A.V., Potapov A.S. Concerning one peculiarity of the MHD-wave field in an inhomogeneous plasma. Issledobaniya po geomagnetizmu, aeronomii i fizike Solntsa [Res. on Geomagnetism, Aeronomy and Solar Physics]. 1984, vol. 70, pp. 149-157. (In Russian).

Golovchanskaya I.V., Kullen A., Maltsev Y.P., Biernat H. Ballooning instability at the plasma sheet-lobe interface and its implications for polar arc formation. J. Geophys. Res. 2006, vol. 111, no. A11216. DOI: 10.1029/2005JA011092.

Hasegawa A. Drift mirror instability of the magnetosphere. Physics of Fluids. 1969, vol. 12, pp. 2642-2650. DOI: 10.1063/1.1692407.

Kaneko T., Goossens M., Soler R., Terradas J., Van Doorsselaere T., Yokoyama T., Wright A.N. Apparent crossfield superslow propagation of magnetohydrodynamic waves in solar plasmas. The Astrophysical J. 2015, vol. 812, no. 2, pp. 2369-2375. DOI: 10.1088/0004-637X/812/2/121.

Klimushkin D.Yu. Spatial structure of small-scale azimuthal hydromagnetic waves in an axisymmetric magnetospheric finite pressure plasma. Plasma Phys. Rep. 1997, vol. 23, pp. $858-871$.

Klimushkin D.Yu. Theory of azimuthally small-scale hydromagnetic waves in the axisymmetric magnetosphere with finite plasma pressure. Ann. Geophys. 1998, vol. 16, pp. 303-321.

Klimushkin D.Yu., Mager P.N., Glassmeier K.H. Toroidal and poloidal Alfvén waves with arbitrary azimuthal wave numbers in a finite pressure plasma in the Earth's magnetosphere. Ann. Geophys. 2004, vol. 22, pp. 267-288. DOI: 10.5194/angeo-22-267-2004.

Klimushkin D.Yu., Mager P.N., Pilipenko V.A. On the ballooning instability of the coupled Alfvén and drift compressional modes. Earth, Planets and Space. 2012, vol. 64, pp. 777-781. DOI: 10.5047/eps.2012.04.002.

Kostarev D.V., Mager P.N. Drift-compression waves propagating in the direction of energetic electron drift in the magnetosphere. Solar-Terr. Phys. 2017, vol. 3, iss. 3, pp. 18-27. DOI: 10.12737/stp-33201703.

Leonovich A.S., Mazur V.A. A theory of transverse smallscale standing Alfvén waves in an axially symmetric magnetosphere. Planetary Space Sci. 1993, vol. 41, pp. 697-717. DOI: 10.1016/0032-0633(93)90055-7.

Leonovich A.S., Klimushkin D.Yu., Mager P.N. Experimental evidence for the existence of monochromatic transverse small-scale standing Alfvén waves with spatially dependent polarization. J. Geophys. Res.: Space Phys. 2015, vol. 120, pp. 5443-5454. DOI: 10.1002/2015JA021044.

Liu W.W. Physics of the explosive growth phase: Ballooning instability revisited. J. Geophys. Res. 1997, vol. 102, no. A3, iss. 2156-2202, pp. 4927-4931. DOI: 10.1029/ 96JA03561. 
Mager P.N., Klimushkin D.Yu. Generation of Alfvén waves by a plasma inhomogeneity moving in the Earth's magnetosphere. Plasma Phys. Rep. 2007, vol. 33, pp. 391-398. DOI: 10.1134/S1063780X07050042.

Mager P.N., Klimushkin D.Yu. Non-resonant instability of coupled Alfvén and drift compressional modes in magnetospheric plasma. Plasma Physics and Controlled Fusion. 2017, vol. 59, no. 9, p. 095005 DOI: 10.1088/1361-6587/aa790c.

Mager P.N., Mikhailova O.S., Mager O.V., Klimushkin D.Yu.. Eigenmodes of the transverse Alfvénic resonator at the plasmapause: A Van Allen probes case study. Geophys. Res. Lett. 2018, vol. 45, pp. 10,796-10,804. DOI: 10.1029/ 2018GL079596.

Mazur N.G., Fedorov E.N., Pilipenko V.A. Dispersion relation for ballooning modes and condition of their stability in the near-Earth plasma. Geomagnetism and Aeronomy. 2012, vol. 52, pp. 603-612.

Mazur V.A., Chuiko D.A. Kelvin-Helmholtz instability on the magnetopause, magnetohydrodynamic waveguide in the outer magnetosphere, and Alfvén resonance deep in the magnetosphere. Plasma Phys. Rep. 2013, vol. 39, no. 6, pp. 488-503.

McPherron R.L. Magnetic pulsations: Their sources and relation to solar wind and geomagnetic activity // Surveys in Geophysics. 2005. V. 26. P. 545-592. DOI: 10.1007/s10712-0051758-7.

Mikhailovskii A.B., Fridman A.M. Drift waves in a finitepressure plasma. Soviet J. Experimental and Theoretical Phys. 1967, vol. 24, pp. 965-974.

Moiseev A.V., Baishev D.G., Mullayarov V.A., et al. The development of compression long-period pulsations on the recovery phase of the magnetic storm on May 23, 2007. Cosmic Res. 2016, vol. 54, pp. 31-39. DOI: 10.1134/S0010952516010123.

Moore T.E., Gallagher D.L. MHD wave breaking in the outer plasmasphere. Geophys Res. Lett. 1987, vol. 14, no. 10 , pp. 1007-1010.

Pokhotelov O.A., Pilipenko V.A., Amata E. Drift anisotropy instability of a finite-beta magnetospheric plasma. Planet Space Sci. 1985, vol. 33, pp. 1229-1241. DOI: $10.1016 / 0032$ 0633(85)90001-7.

Rae I.J., Murphy K.R., Watt C.E.J., Rostoker G., Rankin R., Mann I.R. Field line resonances as a trigger and a tracer for substorm onset. J. Geophys. Res.: Space Phys. 2014, vol. 119, no. 1, pp. 5343-5363. DOI: 10.1002/2013JA018889.

Rosenbluth M.N. Magnetic trapped-particle modes. Phys. Rev. Lett. 1981, vol. 46, pp. 1525-1528. DOI: 10.1103/ PhysRevLett.46.1525.

Rubtsov A.V., Agapitov O.V., Mager P.N., Klimushkin D.Yu., Mager O.V., Mozer F.S., Angelopoulos V. Drift resonance of compressional ULF waves and substorm-injected protons from multipoint THEMIS measurements. J. Geophys. Res.: Space Phys. 2018a, vol. 123, no. 11, pp. 9406-9419. DOI 10.1029/2018JA025985.

Rubtsov A.V., Mager P.N., Klimushkin D.Yu. Ballooning instability of azimuthally small scale coupled Alfvén and slow magnetoacoustic modes in two-dimensionally inhomogeneous magnetospheric plasma. Physics of Plasmas. 2018b, vol. 25, no. 10, 102903 p. DOI: $10.1063 / 1.5051474$.

Southwood L. Some features of field line resonances in the magnetosphere. Planet. Space Sci. 1974, vol. 22, pp. 483-491. DOI: 10.1016/0032-0633(74)90078-6.

Southwood D.J., Saunders M.A. Curvature coupling of slow and Alfvén MHD waves in a magnetotail field configuration. Planet. Space Sci. 1985, vol. 33, pp. 127-134. DOI 10.1016/0032-0633(85)90149-7.

Takahashi K., Denton R.E., Motoba T., et al. Impulsively
Excited Nightside Ultralow Frequency Waves Simultaneously Observed on and off the Magnetic Equator. J. Geophys. Res.: Space Phys. 2018, vol. 45, no. 16, pp. 7918-7926. DOI: 10.1029/2018GL078731.

Ukhorskiy A.Y., Sitnov M.I., Takahashi K., Anderson B.J. Radial transport of radiation belt electrons due to stormtime Pc5 waves. Ann. Geophys. 2009, vol. 27, pp. 2173-2181. DOI: 10.5194/angeo-27-2173-2009.

Walker A.D.M. Theory of magnetospheric standing hydromagnetic waves with large azimuthal wave number, 1 , Coupled magnetosonic and Alfvén waves. J. Geophys. Res. 1996, vol. 101, pp. 27133-27148. DOI: 10.1029/96JA02701.

Woch J., Kremser G., Korth A., Pokhotelov O.A., Pilipenko V.A., Nezlina Yu.M., Amata E. Curvature-driven drift mirror instability in the magnetosphere. 1988, vol. 36, pp. 383-393. DOI: 10.1016/0032-0633(88)90126-2.

Yumoto K. Characteristics of localized resonance coupling oscillations of the slow magnetosonic wave in a non-uniform plasma. Planet. Space Sci. 1985, vol. 33, pp. 1029-1036. DOI: 10.1016/0032-0633(85)90021-2.

Zolotukhina N.A. Excitation of Alfvén waves in the magnetosphere by a moving source. Issledobaniya po geomagnetizmu, aeronomii i fizike Solntsa [Res. on Geomagnetism, Aeronomy and Solar Physics]. 1974, vol. 34, pp. 20-23. (In Russian).

How to cite this article

Petrashchuk A.V., Klimushkin D.Yu. Spatial structure of azimuthally small-scale MHD waves in one-dimensionally inhomogeneous finite pressure plasma with curved field lines. Solar-Terrestrial Physics. 2020. Vol. 6. Iss 1. P. 50-59. DOI: 10.12737/stp-61202006. 